\title{
AVALIAÇÃO DO COMPORTAMENTO DE SEMENTES DE ALGODOEIRO (Gossypium hirsutum L.) DURANTE O ARMAZENAMENTO *
}

\author{
Claudio Bragantini ** \\ Julio Marcos Filho $* * *$ \\ JaIro T. M. Abrahão $* * *$ \\ RoDOLFO GoDoY
}

RESUMO

Sementes de algodoeiro, variedade IAC 13-1, foram armazenadas em câmara seca e em condições normais de ambiente, durante oito meses.

Periodicamente, com intervalos bimestrais, foi avaliado o comportamento das sementes das duas porções, através de testes de germinação e de vigor (envelhecimento rápido e irradiação de sementes), no Laboratório de Sementes do Departamento de Agricultura e Horticultura, ESALQ, USP.

As análises dos dados e a interpretação dos resultados permitiram concluir que:

a) As sementes conservaram-se melhor em câmara seca.

b) Há possibilidade do emprego de irrađiação para avaliar o vigor de sementes, obtendo-se resultados comparáveis aos do envelhecimento rápido, dependendo da dosagem utilizada.

\section{INTRODUÇAO}

As sementes de algodoeiro (Gossypium hirsutum L.) são colhidas no Estado de São Paulo, principalmente nos meses de março, abril e maio e conservadas até outubro-novembro, quando são utilizadas para semeadura.

Sendo ricas de óleo $(25,0-40,0 \%)$, exigem cuidados especiais durante o período de conservação para que mantenham suas boas qualidades. No entanto, durante esse período, normalmente ocorre a deterioração, em ve-

* Entregue para publicação em 26/08/1974.

* Engo Agro - Convênio Ministério da Agricultura/AGIPLAN/USP.

*** Depto. de Agricultura e Horticultura - ESALQ - USP. 
locidade e intensidade variáveis de acordo com o estado fisiológico das sementes e com as condições ambientais.

Diversos pesquisadores, dentre os quais MERCADO (1967), verificaram que os testes de vigor são mais indicados que o teste padråo de germinação para a avaliação do estágio de deterioração das sementes; entre os testes de vigor, um dos mais recomendados é o envelhecimento rápido efetuado em laboratório.

0 presente trabalho visa verificar o comportamento de sementes de algodoeiro quando conservados em dois ambientes diferentes e sua avaliação através de testes de germinação e de vigor. Visa, também, verificar a possibilidade de emprego da irradiação de sementes para avaliar seu estágio de deterioração.

\section{REVISÃO DE LITERATURA}

Muitos pesquisadores, estudando os fatores que afetam a conservação das sementes de diversas espécies cultivadas, concluiram que as condiçōes ambientais durante o período de armazenamento e o teor de umidade das sementes são fatores que afetam diretamente a longevidade.

SIMPSON (1935), trabalhando com sementes de algodoeiro, raça Latifolium, observou que teores de umidade acima de $10 \%$ prejudicaram a conservação das sementes; constatou, também, baixos níveis de deterioração, em sementes conservadas com teores de umidade inferiores a $9 \%$ durante quatro anos. Resultados semelhantes foram constatados por SIMPSON e MILLNER (1944).

Dando continuidade aos seus estudos com sementes de algodoeiro, SIMPSON (1953) armazenou sementes com diversos teores de umidade, entre 7,0 e $14 \%$, em ambientes com temperaturas constantes de $32^{\circ} \mathrm{C}, 21^{\circ} \mathrm{C}$ e $0,5^{\circ} \mathrm{C}$. As sementes armazenadas a $32^{\circ} \mathrm{C}$ apresentaram rápida deterioração e as armazenadas com $14 \%$ de umidade estavam todas mortas após 4 meses. As grandes diferenças de longevidade, influenciadas pelos diferentes teores de umidade da semente e pelas temperaturas do ambiente de armazenamento, forneceram provas conclusivas da relação entre a umidade, a temperatura e a deterioração de sementes.

MERCADO (1967), procurando estudar o comportamento de sementes de algodoeiro conservadas em ambientes com diferentes níveis de umidade relativa verificou, após 13 meses, um declínio de $35 \%$ no poder germinativo das sementes armazenadas com $11-12 \%$ de umidade (ambiente com $75 \%$ UR); não observou queda no poder germinativo em sementes conservadas com 7-8\% de umidade (ambiente com 35\% UR). Por outro lado, os testes de vigor revelaram queda mais acentuada da qualidade das sementes, embora a umidade relativa de $35 \%$ tenha favorecido a conservação.

Sendo o teste de germinação muitas vezes insuficiente para estimar a qualidade de um lote de sementes, diversos pesquisadores passaram a recomendar 
testes de vigor para avaliar o estado fisiológico das sementes. TOLEDO (1966), trabalhando com sementes de algodoeiro, comparou métodos de laboratório para a determinação do vigor e observou que o teste de envelhecimento rápido era promissor para a espécie. Resultados semelhantes foram obtidos por PILI (1966), concluindo que os resultados desse teste são estreitamente associados com os resultados de armazenamento em diferentes condições de ambiente.

Comparando diferentes métodos para determinar o vigor de sementes de milho, WOODSTOCK e COMBS (1964) concluiram que o desenvolvimento da parte aérea das plântulas forneceu as melhores indicações sobre o vigor das sementes. Procuraram, também aplicar diferentes doses de radiações gama às sementes objetivando associar seus efeitos com o vigor, mas não obtiveram resultados satisfatórios.

As aplicações de radiações gama em sementes tem sido estudadas principalmente sob o ponto de vista genético; poucos autores tem se preocupado em verificar seus efeitos sobre a qualidade das sementes.

MC KAY e GOODSPEED, citados por SHULL e MITCHELL (1933), verificaram que as radiações ionizantes aplicadas a sementes de algodoeiro podem promover efeitos letais e tendências à esterilidade das plantas.

OSBORNE e BACON (1960) observaram que doses superiores à $10 \mathrm{krad}$ de radiações gama prejudicaram sensivelmente a germinação e 0 desenvolvimento das plantas de algodoeiro.

OSBORNE e LUNDEN (1961)constataram que a dose de $21 \mathrm{krad}$ de radiações gama reduziu de $50 \%$ a porcentagem de germinação dessas sementes, em relação às não irradiadas.

Por outro lado, YOUNIS e HAMMOUDA (1961), verificaram que a dose necessária para impedir a germinação de 50\% das sementes de algodoeiro, de diversas amostras, foi de $3,4 \mathrm{krad}$ e que todas as doses pesquisadas, de 1,1 a 26,0 krad prejudicaram o poder germinativo.

\section{MATERIAL E MÉTODO}

\section{Sementes}

As sementes destinadas a instalação dos testes, da variedade IAC 13-1, com $98 \%$ de pureza e $80 \%$ de germinação foram obtidas na Secção de Algodão do Instituto Agronômico do Estado de São Paulo (CIA), em Campinas. Após deslintamento químico com ácido sulfúrico concentrado e lavagem om água corrente, foram colocadas em câmara seca para uniformização do teor de umidade.

\section{Ambientes de Conservação}

O material deslintado e com teor de umidade uniforme (7,3\%) foi dividido em duas porções iguais e conservado em dois ambientes diferentes: 
em câmara seca, do Laboratório de Sementes do Departamento de Agricultura e Horticultura, ESALQ, USP, com umidade relativa de $35 \%$ e temperatura média de $25^{\circ} \mathrm{C}\left(\mathrm{A}_{1}\right)$ e em condições normais de ambiente do referido laboratório $\left(\mathrm{A}_{2}\right)$, sendo anotadas a umidade relativa e temperatura do ar através de termohigrógrafo; as médias diárias foram, posteriormente, calculadas de acordo com o método recomendado pelo Serviço Meteorológico Estadual, SP.. As referidas médias encontram-se nas tabelas I e II.

\section{Periodo Experimental}

As sementes foram conservadas nos ambientes citados, de fevereiro a outubro de 1973, perfazendo um total de 8 meses. Periodicamente, com intervalos bimestrais, avaliou-se os efeitos dos tratamentos, através de testes de germinação instalados em quatro épocas, a saber:

$\mathrm{E}_{1}$ fevereiro de 1973; $\mathrm{E}_{2}=$ abril de 1973; $\mathrm{E}_{3}=$ junho de $1973 ; \mathrm{E}_{4}=$ agosto de 1973 e $\mathrm{E}_{5}$ : = outubro de 1973.

\section{Teor de Umidade das Sementes}

Previamente à instalação de cada um dos testes de germinação, foram determinados os teores de umidade das sementes, segundo as Regras para a Análise de Sementes (MINISTÉRIO DA AGRICULTURA, 1967). Os resultados obtidos são apresentados no quadro $\mathrm{I}$.

Quadro I - Teores de umidade das sementes conservadas em dois ambeintes: dados de seis épocas bimestrais (\%).

\begin{tabular}{|c|c|c|}
\hline \multirow{2}{*}{ Épocas } & \multicolumn{2}{|c|}{ Ambientes } \\
\cline { 2 - 3 } & $\mathbf{A}_{1}$ & $\mathbf{A}_{2}$ \\
\hline $\mathrm{E}_{1}$ & 7,3 & 7,3 \\
$\mathrm{E}_{2}$ & 7,0 & 11,1 \\
$\mathrm{E}_{3}$ & 7,4 & 11,4 \\
$\mathrm{E}_{4}$ & 7,4 & 10,7 \\
$\mathrm{E}_{5}$ & 7,1 & 10,6 \\
\hline
\end{tabular}

\section{Testes de Germinação}

Os testes de germinação foram efetuados em germinador BURROWS, a $30^{\circ} \mathrm{C}$ e $95-100 \%$ de umidade relativa. Utilizou-se papel-toalha XUGA, previamente lavado em água corrente durante 24 horas, como substrato.

Cada teste foi instalado com 4 repetições de 50 sementes e as interpretações efetuadas no $5 .^{\circ}$ dia após sua instalação. 


\section{Tratamentos}

0 material armazenado em cada um dos ambientes foi dividido em seis (6) amostras; cinco dessas amostras foram submetidas a diferentes doses de radiações gama de ${ }^{60} \mathrm{Co}$, em fevereiro de 1973, no Centro de Energia Nuclear na Agricultura, ESALQ, USP. As doses de irradiação utilizadas foram as seguintes: $16,0 \mathrm{krad}$.

$\mathrm{T}_{0}=$ testemunha; $\mathrm{T}_{1}=4,0 \mathrm{krad} ; \mathrm{T}_{2}=8,0 \mathrm{krad} ; \mathrm{T}_{3}=12,0 \mathrm{krad} ; \mathrm{T}_{4}=$

A amostra restante foi dividida em cinco sub-amostras; estas foram submetidas ao teste de envelhecimento precoce, conforme descrição de TOLEDO (1966), previamente à instalação de cada um dos testes de germinação, constituindo este, 0 tratamento $T_{5}$.

\section{Métodos Estatísticos}

Os dados de germinação, transformados em arc sen $\sqrt{ } \%$ (SNEDECOR, 1945), foram submetidos a análise estatística das cinco épocas em conjunto, segundo esquema fatorial.

As médias foram comparadas pelo método de Tukey. 
Tabela I: Conservação em condições normais de ambiente: médlas diárias de umidade relativa do ar (\%).

\begin{tabular}{|c|c|c|c|c|c|c|c|c|}
\hline Dia & FEV & MAR & ABR & MAI & JUN & JUL & AGO & SET \\
\hline 1 & 62,8 & 63,5 & 75,0 & 69,8 & 72,0 & 77,8 & 57,2 & 60,5 \\
\hline 2 & 63,5 & 62,5 & 76,3 & 68,5 & 72,0 & 82,8 & 61,2 & 65,8 \\
\hline 3 & 65,3 & 63,5 & 78,3 & 70,8 & 73,0 & 80,0 & 58,0 & 72,5 \\
\hline 4 & 66,3 & 66,5 & 75,5 & 70,3 & 75,5 & 80,5 & 64,0 & 72,5 \\
\hline 5 & 67,3 & 65,3 & 71,5 & 67,0 & 73,0 & 79,0 & 71,0 & 65,2 \\
\hline 6 & 63,5 & 60,0 & 74,3 & 63,3 & 71,0 & 72,3 & 73,2 & 71,0 \\
\hline 7 & 58,3 & 58,3 & 71,5 & 68,0 & 68,5 & 77,8 & 65,5 & 59,8 \\
\hline 8 & 54,5 & 72,0 & 72,0 & 76,0 & 68,5 & 73,5 & 66,2 & 64,0 \\
\hline 9 & 58,3 & 68,8 & 69,0 & 71,0 & 68,5 & 66,5 & 59,5 & 67,0 \\
\hline 10 & 60,3 & 63,8 & 65,0 & 59,5 & 72,8 & 65,3 & 57,7 & 72,0 \\
\hline 11 & 58,3 & 63,3 & 68,3 & 54,3 & 73,5 & 63,8 & 62,5 & 68,2 \\
\hline 12 & 63,0 & 61,5 & 72,0 & 57,0 & 72,8 & 65,0 & 65,8 & 64,2 \\
\hline 13 & 65,0 & 58,0 & 76,0 & 62,5 & 60,3 & 62,8 & 56,5 & 63,0 \\
\hline 14 & 68,5 & 57,2 & 78,0 & 59,0 & 69,5 & 64,8 & 64,5 & 64,5 \\
\hline 15 & 77,5 & 59,5 & 76,8 & 58,5 & 72,0 & 64,0 & 67,0 & 65,2 \\
\hline 16 & 80,0 & 62,0 & 75,3 & 60,0 & 74,0 & 66,5 & 60,8 & 66,0 \\
\hline 17 & 78,3 & 65,7 & 70,3 & 67,0 & 71,0 & 62,8 & 52,8 & 63,8 \\
\hline 18 & 73,8 & 62,5 & 71,5 & 67,8 & 73,0 & 69,5 & 58,0 & 57,0 \\
\hline 19 & 69,0 & 61,0 & 72,5 & 69,5 & 54,3 & 71,3 & 58,5 & 54,0 \\
\hline 20 & 68,5 & 68,0 & 70,8 & 73,8 & 60,3 & 63,5 & 62,8 & 52,2 \\
\hline 21 & 72,0 & 73,0 & 73,0 & 72,5 & 64,0 & 64,5 & 62,8 & 57,5 \\
\hline 22 & 63,8 & 76,0 & 73,5 & 78,0 & 70,3 & 58,5 & 59,2 & 57,5 \\
\hline 23 & 58,3 & 67,5 & 70,8 & 79,0 & 73,0 & 60,3 & 63,5 & 57,5 \\
\hline 24 & 61,0 & 63,8 & 69,3 & 77,8 & 75,8 & 58,0 & 63,5 & 61,8 \\
\hline 25 & 61,5 & 65,5 & 65,5 & 73,3 & 78,5 & 52,5 & 61,5 & 67,8 \\
\hline 26 & 64,5 & 62,5 & 69,3 & 72,0 & 72,3 & 56,0 & 67,2 & 71,8 \\
\hline 27 & 70,0 & 42,0 & 69,3 & 70,3 & 83,0 & 58,8 & 62,5 & 75,2 \\
\hline 28 & 62,8 & 34,0 & 72,5 & 68,8 & 75,0 & 62,3 & 65,2 & 66,2 \\
\hline 29 & - & 58,0 & 70,3 & 72,3 & 63,5 & 59,5 & 56,2 & 61,0 \\
\hline 30 & - & 76,0 & 72,8 & - & 72,3 & 58,5 & 58,0 & 59,2 \\
\hline 31 & - & 76,5 & - & - & - & 61,0 & 61,2 & - \\
\hline Média & 65,9 & 63,2 & 72,2 & 68,2 & 70,8 & 66,41 & 62,1 & 64,1 \\
\hline
\end{tabular}


Tabola II: Conservação em condições normais de ambiente: temperatura média diária $1{ }^{\circ} \mathrm{C}$.

\begin{tabular}{|c|c|c|c|c|c|c|c|c|}
\hline Dia & FEV & MAR & ABR & MAI & JUN & JUL & AGO & SET \\
\hline 1 & 27,2 & 27,7 & 24,7 & 23,6 & 20,3 & 19,4 & 17,2 & 19,0 \\
\hline 2 & 27,8 & 27,4 & 25,1 & 23,2 & 19,8 & 20,2 & 18,0 & 17,9 \\
\hline 3 & 27,9 & 27,1 & 25,2 & 23,3 & 20,1 & 20,0 & 18,6 & 18,5 \\
\hline 4 & 28,1 & 26,8 & 25,7 & 23,3 & 20,7 & 19,7 & 17,8 & 19,3 \\
\hline 5 & 27,9 & 26,8 & 26,2 & 23,2 & 21,0 & 19,6 & 17,8 & 20,0 \\
\hline 6 & 27,8 & 26,3 & 26,3 & 22,8 & 21,1 & 19,4 & 18,3 & 20,4 \\
\hline 7 & 26,8 & 26,3 & 26,7 & 22,4 & 21,6 & 19,7 & 17,9 & 19,1 \\
\hline 8 & 25,4 & 26,2 & 26,4 & 21,9 & 21,9 & 20,3 & 17,9 & 18,1 \\
\hline 9 & 25,8 & 25,6 & 27,2 & 22,1 & 22,1 & 20,1 & 18,3 & 18,8 \\
\hline 10 & 27,2 & 24,8 & 26,1 & 20,4 & 22,6 & 20,5 & 19,3 & 20,4 \\
\hline 11 & 27,5 & 24,8 & 25,1 & 18,1 & 22,1 & 20,5 & 19,8 & 21,1 \\
\hline 12 & 28,5 & 24,7 & 24,5 & 17,4 & 20,8 & 21,1 & 20,1 & 22,4 \\
\hline 13 & 27,4 & 25,6 & 24,7 & 17,6 & 20,2 & 21,5 & 20,7 & 22,6 \\
\hline 14 & 27,0 & 25,3 & 25,2 & 17,8 & 20,5 & 21,0 & 22,4 & 23,5 \\
\hline 15 & 26,2 & 25,3 & 25,8 & 18,2 & 20,5 & 20,4 & 21,2 & 23,6 \\
\hline 16 & 26,0 & 26,2 & 25,8 & 18,7 & 20,2 & 21,2 & 20,4 & 23,6 \\
\hline 17 & 26,0 & 26,7 & 26,0 & 19,6 & 20,2 & 23,9 & 18,7 & 24,0 \\
\hline 18 & 26,4 & 27,2 & 26,0 & 20,1 & 20,7 & 22,5 & 18,6 & 24,4 \\
\hline 19 & 27,6 & 26,9 & 26,2 & 19,6 & 17,5 & 21,6 & 18,8 & 24,4 \\
\hline 20 & 26,7 & 26,8 & 25,6 & 20,2 & 16,0 & 20,8 & 19,7 & 23,0 \\
\hline 21 & 26,5 & 26,4 & 25,2 & 21,5 & 15,4 & 21,0 & 21,0 & 21,4 \\
\hline 22 & 27,4 & 26,1 & 22,0 & 20,7 & 16,5 & 19,3 & 20,3 & 20,4 \\
\hline 23 & 27.7 & 25,1 & 24,3 & 20,4 & 18,1 & 18,2 & 20,3 & 19,9 \\
\hline 24 & 27,5 & 24,8 & 24,0 & 20,7 & 19,3 & 17,8 & 21,0 & 20,8 \\
\hline 25 & 27,1 & 24,8 & 24,0 & 21,5 & 20,3 & 16,9 & 21,7 & 21,5 \\
\hline 26 & 27,8 & 25,2 & 24,7 & 21,4 & 18,9 & 17,1 & 22,2 & 21,1 \\
\hline 27 & 27,5 & 25,7 & 24,1 & 21,8 & 19,7 & 17,8 & 21,4 & 21,4 \\
\hline 28 & 27,5 & 26,1 & 24,1 & 21,8 & 19,1 & 17,9 & 20,1 & 21,8 \\
\hline 29 & - & 25,8 & 24,3 & 21,8 & 17,6 & 16,5 & 19,0 & 21,3 \\
\hline 30 & - & 24,4 & - & 21,5 & 17,8 & 16,1 & 19,0 & 21,0 \\
\hline 31 & - & 24,3 & - & 20,8 & - & 16,4 & 19,3 & - \\
\hline MÉdia & 27,12 & 25,04 & 25,18 & 20,21 & 19,75 & 19,63 & 19,57 & 21,96 \\
\hline
\end{tabular}

\section{RESULTADOS}

A análise da variância dos dados obtidos nos testes de germinação, revelou valores de $\mathrm{F}$ significativos, ao nível de $1 \%$ de probabilidade, para os efeitos de tratamentos, de épocas de ambientes e para interação épocas $\mathrm{x}$ ambientes, conforme mostra o Quadro IV. 
Quadro IV: Análise da variância dos dados de germinação $(x=\operatorname{arc}$ sen $\sqrt{ } \%)$.

\begin{tabular}{|l|r|r|r|c|}
\hline Causas de Variação & G.L. & \multicolumn{1}{|c|}{ S.Q. } & OM. & F \\
\hline Tratamentos (T) & 5 & 6716,08 & $1.343,22$ & $102,07 * *$ \\
Epocas (E) & 4 & 11380,42 & $2.845,10$ & $216,19 * *$ \\
Ambiente (A) & 1 & 769,52 & 769,52 & $58,47 * *$ \\
T X E & 20 & 942,14 & 47,11 & 3,58 \\
T X A & 5 & 35,38 & 7,08 & 0,54 \\
EXA & 4 & 237,16 & 59,29 & $4,51 * *$ \\
Residuo & 200 & 2631,20 & 13,16 & \\
\hline TOTAL & 239 & 22711,90 & & \\
\hline
\end{tabular}

\section{Efeitos de Tratamentos}

No Quadro $\mathrm{V}$ encontram-se as médias obtidas para os efeitos dos tratamentos, a diferença mínima significativa e o coeficiente de variação:

Quadro V: Germinação de sementes de algodoeiro: Médias obtidas para os efeitos de tratamentos $(x=\operatorname{arcsen} \sqrt{ } \%)$.

\begin{tabular}{|cccccc|}
\hline$T_{0}$ & $\mathbf{T}_{\mathbf{1}}$ & $\mathbf{T}_{\mathbf{2}}$ & $\mathbf{T}_{\mathbf{3}}$ & $\mathbf{T}_{\mathbf{4}}$ & $\mathbf{T}_{\mathbf{5}}$ \\
\hline 54,94 & 52,89 & 48,25 & 44,06 & 39,93 & 52,26 \\
\hline D.M.S. - Tukey (5\%) & & & & 2,6 \\
\hline Coeficiente de Variação & & & & $7,4 \%$ \\
\hline
\end{tabular}

0 exame desse quadro permite verificar que as sementes não irradiadas $\left(\mathrm{T}_{0}\right)$, as submetidas a $4,0 \mathrm{krad}\left(\mathrm{T}_{1}\right)$ e as envelhecidas $\left(\mathrm{T}_{5}\right)$ apresentaram poder germinativo estatísticamente superior ao das submetidas às doses de $8,0 \operatorname{krad}\left(\mathrm{T}_{2}\right), 12,0 \mathrm{krad}\left(\mathrm{T}_{3}\right)$ e $16,0 \mathrm{krad}\left(\mathrm{T}_{4}\right)$.

Por outro lado, as sementes correspondentes ao tratamento com 4,0 krad não apresentam diferenças significativas em relação à testemunha $\left(\mathrm{T}_{0}\right) \mathrm{e}$ às sementes envelhecidas $\left(T_{5}\right)$. A germinação das envelhecidas $\left(T_{5}\right)$ foi significativamente inferior à da testemunha.

\section{Efeitos da interação épocas $x$ ambientes}

As médias, a diferença mínima significativa e o coeficiente de variação referentes aos efeitos dessa interação são encontrados no Quadro VI. 
Quadro VI: Germinação de sementes de algodoeiro: médias obtidas para interação épocas $x$ ambientes $(x=$ arc sen $\sqrt{ } \%)$.

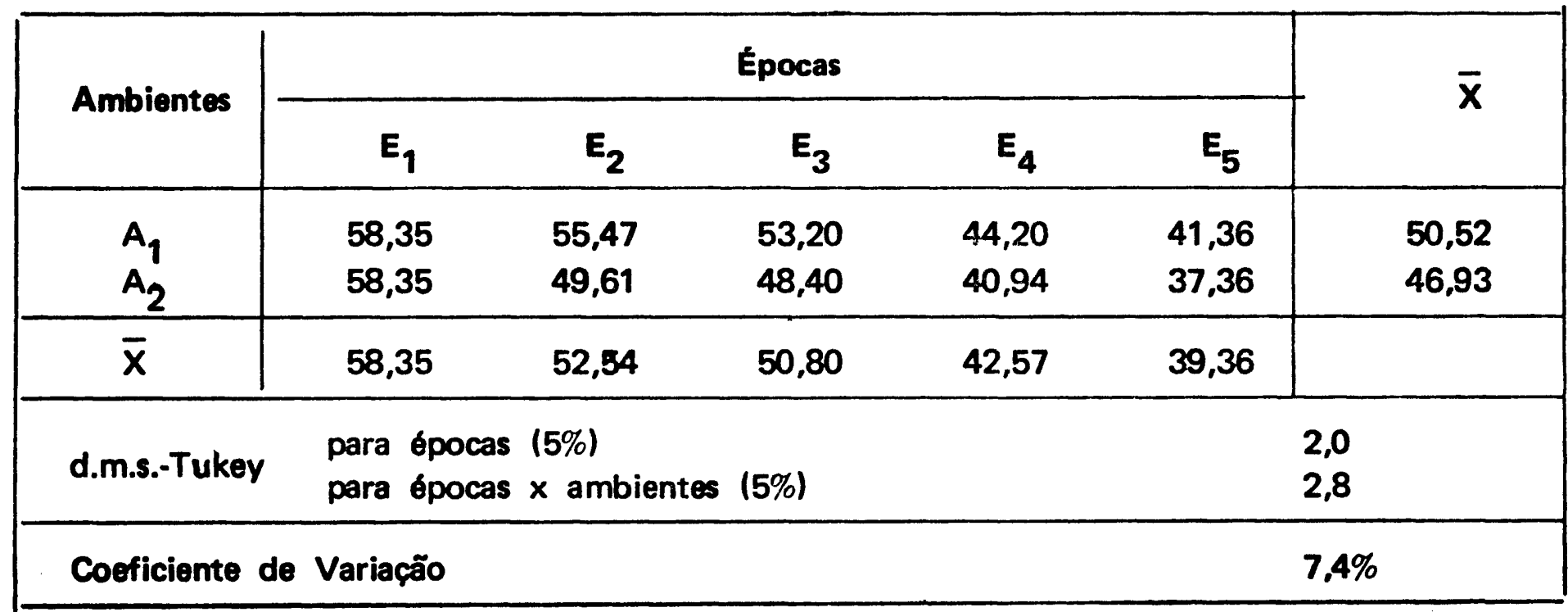

O exame do Quadro VI permite observar que houve queda do poder germinativo com o decorrer do período de conservação, dentro dos dois ambientes, embora a diferença entre $\mathrm{E}_{2}$ e $\mathrm{E}_{3}$ não tenha sido significativa. Também, o poder germinativo das sementes conservadas em câmara seca $\left(A_{1}\right)$ foi superior ao das armazenadas em condições normais de ambiente $\left(\mathrm{A}_{2}\right)$; apenas no teste inicial $\left(E_{1}\right)$ não foi observada diferença estatística entre os ambientes.

\section{DISCUSSÁO}

Os resultados obtidos no presente trabalho revelaram diferenças no comportamento de sementes de algodoeiro, dependendo do ambiente utilizado. A conservação em camara seca foi utilizada como um padrão, pois teoricamente é um ambiente ideal para a manutenção do poder germinativo e do vigor das sementes, por período de tempo prolongado. 0 armazenamento em condições normais de ambiente baseou-se no fato de que a deterioração das sementes é sujeita às variações climáticas do ambiente.

A pesquisa efetuada não poderia ter sido assentada em bases mais sólidas, porque a bibliografia sobre a conservação de sementes de algodoeiro é escassa; os poucos trabalhos relacionados com o assunto foram efetuados em outros países.

Analisando-se os resultados obtidos para os efeitos dos tratamentos, verifica-se que os mesmos se comportaram de maneira semelhante durante todas as épocas e ambientes estudados, pois não houve significância estatística para as interações tratamentos $\mathrm{x}$ épocas e tratamentos $\mathrm{x}$ ambientes.

Verificou-se que os tratamentos com $4,0 \mathrm{krad}\left(\mathrm{T}_{1}\right)$ e a testemunha $\left(T_{0}\right)$ foram estatísticamente semelhantes, contrariando os resultados obtidos por YOUNIS e HAMMOUDA (1961) e concordando com as observações de 
OSBORNE e BACON (1960). A maior sensibilidade das sementes submetidas a $12,0 \mathrm{krad}\left(\mathrm{T}_{3}\right)$ e $16,0 \mathrm{krad}\left(\mathrm{T}_{4}\right)$ também foi observada por OSBORNE e BACON (1960).

0 período de envelhecimento empregado como tratamento $\left(T_{5}\right)$, previamente à instalação de cada teste de germinação, foi o mesmo utilizado por TOLEDO (1966) e PILLI (1966) para a avaliação do vigor das sementes. Os resultados mostraram, no presente trabalho, redução do poder germinativo em relação à testemunha $\left(\mathrm{T}_{0}\right)$, acusando um nível superior de deterioração das sementes em relação ao revelado pelo teste de germinação.

Notou-se também que os resultados de envelhecimento foram comparáveis aos do tratamento com $4,0 \mathrm{krad}$; isto parece revelar a possibilidade de se utilizar efeitos de irradiação para avaliar o nível de deterioração das sementes. Isto não foi verificado por WOODSTOCK e COMBS (1964), em sementes de milho, podendo-se dizer que conclusões mais objetivas sobre essa possibilidade somente poderão ser obtidas após estudos mais aprofundados.

A análise da interação épocas $\mathbf{x}$ ambientes revelou a queda do poder germinativo com o decorrer do período de conservação, principalmente para as sementes mantidas em condições normais de ambiente $\left(\mathrm{A}_{2}\right)$. A conservação em câmara seca conduziu a melhores resultados, confirmando as observações de MERCADO (1967). No entanto, este autor não constatou deterioração em sementes conservadas em ambiente com $35 \%$ de umidade relativa, que foi observada no presente trabalho.

Os resultados também confirmaram as conclusões de SIMPSON (1953), segundo as quais a conservação de sementes de algodoeiro é muito influenciada pelas condições do ambiente e pelo teor de umidade das sementes. Revelaram, ainda a sensibilidade das sementes de algodoeiro às condições de armazenamento, pois a deterioração ocorreu mesmo quando as sementes foram conservadas com teores de umidade relativamente baixos, tanto em câmara seca $(7,0-7,4 \%)$ como em condições normais de ambiente (10,6$11,4 \%)$.

\section{CONCLUSÓES}

As análises dos dados e a interpretação dos resultados da presente pesquisa permitiram concluir que:

a) As condições de ambiente que proporcionaram a manutenção de baixos teores de umidade nas sementes, revelaram-se mais favoráveis à conservação.

b) Há possibilidade do emprego de radiações gama para avaliação do estágio de deterioração das sementes,obtendo-se resultados semelhantes aos do envelhecimento rápido, dependendo da dosagem utilizada. 


\section{VIABILITY OF COTTONSEED DURING STORAGE}

\section{SUMMARY}

This study was undertaken to determine the viability of cototn seed during storage under controlled $(35 \% \mathrm{R} . \mathrm{H}$.) and uncontrolled conditions.

Seed germination and two methods of vigor analysis accelerated aging and seed irradiation - were used for evaluation of seed behavior, during eight months.

The results showed that uncontrolled conditions were more detrimental to seed viability. Accelerated aging was found to be an effective method to determine the progress of seed deterioration during storage.

Seed irradiation results may be correlated with accelerated aging, depending on the dosage used.

\section{LITERATURA CITADA}

MERCADO, A. T., 1967 - Moisture equilibrium and quality evaluation of five lrinds of seed stored at various relative humidities M. S. Thesis, Miss. Sta. Univ. : 56 pp.

MINISTERIO DA AGRICULTURA, 1967 - Regras para Análises de Sementes Escritório da Produção Vegetal, Equipe Técnica de Sementes e Mudas : 120 pp.

OSBORNE, T. S. \& J. A. BACON, 1960 - Radiosensitivity of seeds - I. - Reduction or stimulation of seedling grow h as a function of gamma ray dose. Radiation Research 13 (5) : 686-690.

OSBORNE, T. S. \& A. O. LUNDEN, 1961 - The comparative plant and seed irradiation program of the University of Tenessee The International Journal of Applied Radiation and Isotopes 10 (4) : 198-209.

PILLI, E. C., 1966 - An accelerated aging technique for evaluating storabillty of alfafa, wheat, corn and cotton seed lots. M. S: Thesis, Miss. Sta. University.

SHULL, C. A. \& J. W. MITCHELL, 1933 - Stimulative effects of X - Rays - on plant growth. Plant. Physiology 8 (2) : 287-296.

SIMFSON, D. MT., 1935 - Relation of moisture content and method of storage to deterioration of stored cottonseed. Journal of Agricultural Research 50 (7): 449-456.

SIMPSON, D. M. \& P. R. MILLNER, 1944 - The relation of atmospheric humidity to moisture cottonseed. Journal of American Society of Agronomy 36 : 957-959.

SIMPSON, D. M., 1953 - Longevity of cottonseed. Agronomy Journal 45 (8) : 391.

SNEDECOR, G. W., 1945 - Métodos Estatísticos. Ministério da Economia. Lisboa. $469 \mathrm{pp}$.

TOLEDO, F. F., 1966 - Comparação entre métodos de laboratório para a determlnação do vigor em sementes de algodão. Revista de Agricultura 41(1) : 13-16.

WOODSTOCK, L. W. \& M. F. COMBS, 1964 - A comparison of some possib e indices of seedling vigor in corn. Proceedings of the Association of Official Seed Analysts $54: 50-60$.

YOUNIS, A. E. \& M. A. HAMMOUDA, 1961 -- Pre-sowing gamma irradiation of cotton seeds from a point source cf ${ }^{137} \mathrm{Cs}$ and its effect on emergence, growth and yield. Plant and Soil $13(4): 311-321$. 
